\title{
A Class of Preview Control for Dual-Stage Actuator Systems
}

\author{
Aurélio T. Salton, Zhiyong Chen, Minyue Fu
}

\begin{abstract}
This paper proposes a novel "preview control" approach to dual-stage actuator (DSA) systems based on the redundancy of actuators. While conventional tracking controllers are designed such that the primary actuator asymptotically approaches the set point, the proposed methodology allows it to move freely within the range of the secondary actuator. Then, the secondary actuator controller is designed such that the error between the primary actuator and the set point can be compensated. The main advantage of the proposed controller is exhibited in the successive set point control scenario, and its effectiveness is demonstrated by numerical simulation.
\end{abstract}

\section{INTRODUCTION}

Dual-stage actuators (DSA's) servo systems are comprised of two actuators connected in series: a primary actuator characterized by a long travel range but poor accuracy and slow response time; and a secondary actuator of higher precision and faster response, but with limited travel range. An improved performance can be achieved by combining both actuators and making use of an appropriate control strategy, so that the defects of one actuator are compensated by the merits of the other. Many types of DSA's are seen in industrial applications, from hard disk drives (HDDs), pushing up the HDD track density and bandwidth [1] and [2], to dual-stage machine tools [3], XY positioning tables [4] and dual-stage MEMS microactuators [5].

Despite the structural simplicity of DSA's it is a challenge to design controllers for such systems that yield an optimal performance. Due to the redundancy of both actuators, any desired trajectory may be generated by various combinations their outputs. The challenge, therefore, is how to take fully advantage of such redundancy and find an optimal pair of outputs. Most of the work on DSA tracking control is based on designing a primary actuator control loop to yield a small overshoot, and a secondary actuator controller to follow the position error of the primary actuator [6] - [8]. A recent approach in [9] and [10] has showed significant improvement regarding the settling time by allowing the primary actuator to overshoot within the range of the secondary actuator. Yet another approach takes into account the redundancy of the actuators and optimizes the output-transition problem by allowing a post and pre-actuation while guaranteeing the output at a constant value [11].

In order to further reduce the settling time we propose a different approach to dual-stage control design based on the

The authors are with the School of Electrical Engineering and Computer Science, The University of Newcastle, Callaghan, NSW 2308, Australia. This work was supported by the Australian Research Councils Center of Excellence for Complex Dynamic Systems and Control (CDSC). (email: Aurelio.Salton@newcastle.edu.au; Zhiyong.Chen@newcastle.edu.au; Minyue.Fu@newcastle.edu.au). information of future reference points in the successive set point control scenario. It is our belief that it is not necessary for the primary actuator output to asymptotically approach the set point; instead, we allow the primary actuator to move freely in a manifold determined by the range of the secondary actuator. This manifold is constructed such that the reference point is within reach of the secondary actuator, allowing it to compensate the error generated by the primary actuator's movement inside the manifold. Then, we take advantage of the freedom of the primary actuator to optimize the transition time between the current reference point and the next one. A control method that utilizes future information, such as the proposed one, was first introduced in [12] under the name of "preview control".

To present the proposed methodology, the paper is divided into the following sections: Section II formulates the problem and introduces the general idea, the proposed solution is given in Section III, simulation results are presented in Section IV, and Section V concludes the paper.

\section{Problem Formulation}

The general class of DSA treated in this paper is depicted in Fig. 1. Under the assumptions that $M \gg m, y_{1} / y_{2} \gg$ 1 , and $\left|u_{2} / u_{2}\right| \gg m / M$, we may neglect the coupling forces between the primary and the secondary actuators. Furthermore, it is assumed that the friction acting on the primary actuator (if any) is actively compensated by a friction compensator. In this way, we can treat the DSA of interest as a linear decoupled dual-input single-output (DISO) system,

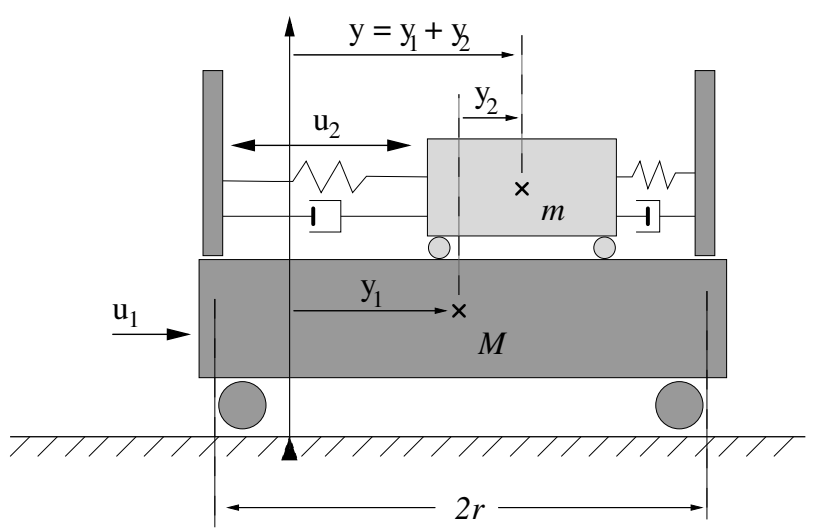

Fig. 1. Schematic representation of the dual-stage actuator (DSA). 
which is represented in a state-space form as:

$$
\begin{aligned}
\Sigma_{1}: \dot{x}_{1} & =A_{1} x_{1}+B_{1} \operatorname{sat}\left(u_{1}\right), \quad x_{1}(0)=0 \\
\Sigma_{2}: \dot{x}_{2} & =A_{2} x_{2}+B_{2} \operatorname{sat}\left(u_{2}\right), x_{2}(0)=0 \\
y & =y_{1}+y_{2}=C_{1} x_{1}+C_{2} x_{2},
\end{aligned}
$$

where $x_{1}=\left[\begin{array}{ll}y_{1} & \dot{y}_{1}\end{array}\right]^{\top}, x_{2}=\left[\begin{array}{ll}y_{2} & \dot{y}_{2}\end{array}\right]^{\top}$,

$$
\begin{gathered}
A_{1}=\left[\begin{array}{ll}
0 & 1 \\
0 & 0
\end{array}\right], B_{1}=\left[\begin{array}{c}
0 \\
b_{1}
\end{array}\right], C_{1}=\left[\begin{array}{ll}
1 & 0
\end{array}\right], \\
A_{2}=\left[\begin{array}{cc}
0 & 1 \\
a_{1} & a_{2}
\end{array}\right], B_{2}=\left[\begin{array}{c}
0 \\
b_{2}
\end{array}\right], C_{2}=\left[\begin{array}{ll}
1 & 0
\end{array}\right],
\end{gathered}
$$

and the constants $a_{1}, a_{2}, b_{1}$, and $b_{2}$ depend on each particular system.

An intrinsic characteristic of such class of systems is that the coarse and fine actuators are complementary to each other: while the primary actuator is slow and has a large travel range, the secondary actuator is fast but has a limited range of actuation. Due to these complementary characteristics of the DSA system we shall make the following assumption,

A1: Within the travel range of the secondary actuator the tracking error of the primary actuator is sufficiently smooth so that it may be followed by the secondary actuator with negligible error.

We would like to propose a novel design strategy for the primary actuator based on Assumption A1. Notice that, when such assumption holds, the purpose of the primary actuator becomes to "carry" the secondary actuator close to the reference, so that the reference is within its travel range.

In other words, if we define a manifold $\boldsymbol{S}_{i}$,

$$
\boldsymbol{S}_{i}=\left\{y_{1} \in \mathbb{R}:\left|y_{1}-r e f_{i}\right| \leq r\right\},
$$

where $r e f_{i}$ is the $i$-th reference point and $\pm r$ is the range of the secondary actuator, then whenever $y_{1}$ is within the manifold $\boldsymbol{S}_{i}$, the total output $y$ of the DSA is at the $i$-th reference point. Therefore, the objective of the primary actuator control is to make $y_{1}$ converge to $\boldsymbol{S}_{i}$ rather than making it asymptotically track the reference (Fig. 2). Furthermore, if the output $y$ must stay at a given initial reference point $r e f_{1}$ for $\tau$ seconds before moving to another given reference $r e f_{2}$, then $y_{1}$ must stay in $\boldsymbol{S}_{1}$ for $\tau$ seconds before moving to $\boldsymbol{S}_{2}$. If the system in hand is a DSA hard-disk drive, for example, we can suppose that from $t=0$ to $t=\tau$ the head of the HDD is reading a given track $r e f_{1}$ and that after it is finished ( $\tau$ seconds) it will move to a different track $r e f_{2}$.

Hence, the problems to be addressed are stated as follows.

P1: For a given initial condition $x_{1}(0)=\left[\begin{array}{ll}p_{0} & v_{0}\end{array}\right]^{\top}$ and two manifolds $\boldsymbol{S}_{1}$ and $\boldsymbol{S}_{2}$ determined by (2), design a controller that drives the output $y_{1}$ of the primary actuator from $\boldsymbol{S}_{1}$ to $\mathrm{S}_{2}$ in a minimum time, under the constraints:

$$
\begin{gathered}
\mathcal{U}_{1} \leq u_{1}(t) \leq \mathcal{U}_{2} \\
y_{1} \in \boldsymbol{S}_{1}, \quad 0 \leq t \leq \tau .
\end{gathered}
$$

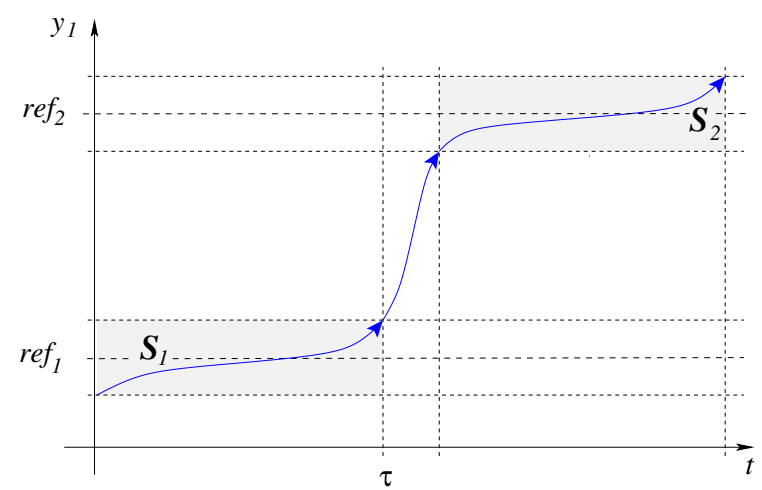

Fig. 2. After $\tau$ seconds in $\boldsymbol{S}_{1}$ the primary must move to $\boldsymbol{S}_{2}$. At $t=\tau$ the output $y_{1}$ should be at the border of $\boldsymbol{S}_{1}$ with the maximal speed towards $S_{2}$.

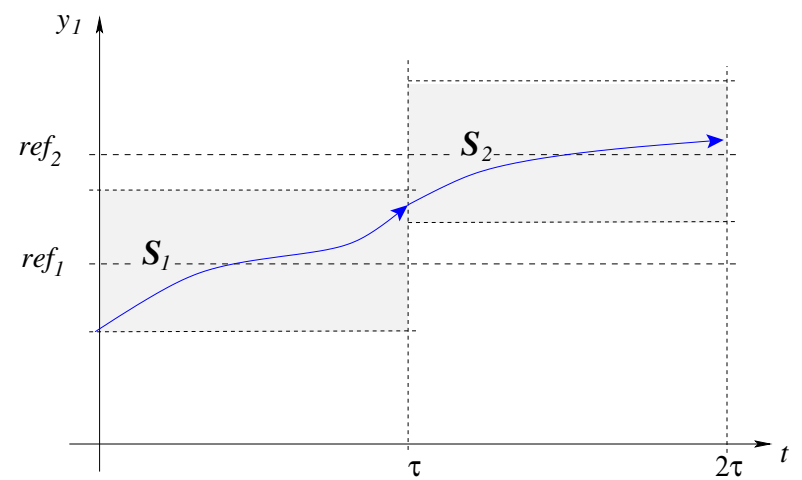

Fig. 3. In the situation where the manifolds $\boldsymbol{S}_{1}$ and $\boldsymbol{S}_{2}$ intersect each other the transition occurs instantly, as long as, at time $t=\tau, y_{1}$ is positioned at the intersection.

P2: Under Assumption A1, when $y_{1} \in S_{i}$, design a controller for the secondary actuator to compensate for the error generated by the primary actuator, i.e., to achieve $y=y_{1}+y_{2}=\operatorname{ref}_{i}$.

\section{Preview Control Design}

In this section we will present the detailed development of the preview control law for the primary actuator responsible for solving P1. We will then expose the control strategy for secondary actuator, responsible for solving $\mathbf{P 2}$. As the main objective of this paper relates to the improvement of the primary actuator control design, we will focus on this matter and borrow an existing controller for the secondary actuator.

\section{A. Primary Actuator Control Design}

The two manifolds $S_{1}$ and $S_{2}$ described in the problem statement may or may not intersect each other. These situations must be treated differently inasmuch as the existence of such intersection allows the instantaneous transfer of the output $y_{1}$ from $\boldsymbol{S}_{1}$ to $\boldsymbol{S}_{2}$. However, when there is no intersection, this transition will not happen instantly and there will exist a transition time which must be minimized in order to improve the performance of the system, (Fig. 2). We shall first expose the former situation, depicted in Fig. 3, where there is an intersection between $\boldsymbol{S}_{1}$ and $\boldsymbol{S}_{2}$. 
1) $\boldsymbol{S}_{1} \cap \boldsymbol{S}_{2} \neq \varnothing$ : Notice that when there is an intersection between the manifolds the transition occurs instantly as long as, at time $t=\tau, y_{1}$ is positioned at both $\boldsymbol{S}_{1}$ and $\boldsymbol{S}_{2}$, as depicted in Fig. 3. This problem may treated as a two-pointboundary optimization problem with the control effort as the cost function. In order to simplify the notation, let us define

$$
p:=y_{1}, \quad v:=\dot{y}_{1}
$$

and suppose, without loss of generality, that $b_{1}=1$. Thus, $x_{1}=\left[\begin{array}{ll}p & v\end{array}\right]^{\top}$, and the primary actuator system's equations are given by,

$$
\dot{x}_{1}=f\left(x_{1}, u_{1}\right)=\left[\begin{array}{ll}
v & u_{1}
\end{array}\right]^{\top} .
$$

In this context the values of $p(0)$ and $v(0)$ are know, and there is a restriction only in $p(\tau)$, which must lie between the manifolds $\boldsymbol{S}_{1}$ and $\boldsymbol{S}_{2}$. Therefore, $v(\tau)$ becomes a design parameter to be chosen to minimize the control effort. The control problem becomes to design a control law, denoted by $u_{1, \tau_{0}}$, that takes the output $y_{1}$ from an initial position $p(0)$ to a final position $p(\tau)$, while minimizing the cost function

$$
J=\frac{1}{2} \int_{t_{0}}^{\tau} u_{1, \tau_{0}}^{2}(t) d t
$$

Proposition 3.1: Given the initial conditions $x_{1}(0)=$ $\left[\begin{array}{ll}p_{0} & v_{0}\end{array}\right]^{\top}$ and final condition $p(\tau)=p_{\tau}$, the optimal input $u_{1, \tau_{0}}$ that minimizes the cost function (6) is given by:

$$
u_{1, \tau_{0}}(t)=a t+b, \quad 0 \leq t \leq \tau
$$

where,

$$
a=3\left(v_{0} \tau-\Delta p\right) / \tau^{3}, \quad b=-\tau a, \quad \Delta p:=p_{\tau}-p_{0} .
$$

Proof: Define $L=u_{1, \tau_{0}}^{2} / 2$ and adjoin the system's equations (5) to the performance index via the Lagrange multipliers $\lambda^{\top}=\left[\begin{array}{ll}\lambda_{1} & \lambda_{2}\end{array}\right]$,

$$
J=\int_{t_{0}}^{\tau} L+\lambda^{\top}\left(f-\dot{x}_{1}\right) d t .
$$

Let $H$ be the Hamiltonian function defined as $H=L+$ $\lambda^{\top} f$, and integrate $\lambda^{\top} \dot{x}_{1}$ by parts to yield,

$$
J=-\left.\lambda^{\top} x_{1}\right|_{0} ^{\tau}+\int_{0}^{\tau}\left(H+\dot{\lambda}^{\top} x_{1}\right) d t
$$

The minimum of $J$ is achieved when $\delta J=0$ [15], therefore,

$$
\begin{aligned}
\delta J= & -\overbrace{\left.\lambda^{\top} \delta x_{1}\right|_{t=\tau}+\left.\lambda^{\top} \delta x_{1}\right|_{t=0}}^{(i)=0} \\
& +\int_{0}^{\tau}\{\underbrace{\left(\frac{\partial H}{\partial x_{1}}+\dot{\lambda}^{\top}\right)}_{(i i)=0} \delta x_{1}+\underbrace{\frac{\partial H}{\partial u_{1, \tau_{0}}} \delta u_{1, \tau_{0}}}_{(i i i)=0}\} d t .
\end{aligned}
$$

The co-states $\lambda$ are of free choice and can be used to simplify the task of computing $\delta J=0$. Hence, $(i)=0$ is achieved by setting

$$
\lambda_{2}(\tau)=0
$$

To achieve $(i i)=0$, simply force

$$
\dot{\lambda}^{\top}=-\frac{\partial H}{\partial x_{1}}=-\frac{\partial L}{\partial x_{1}}-\lambda^{\top} \frac{\partial f}{\partial x_{1}},
$$

and, in order to achieve $(i i i)=0$, one must find a control law $u_{1, \tau_{0}}$ such that

$$
\frac{\partial H}{\partial u_{1, \tau_{0}}}=0 .
$$

The control law (7) is found by solving equations (9), (10) and (11), while respecting the constraints $y_{1}=p(0)$ for $t=0$ and $y_{1}=p(\tau)$ for $t=\tau$.

2) $S_{1} \cap S_{2}=\varnothing$ : In this situation, depicted in Fig. 2, the control objective is to guarantee $y_{1}$ will stay within $\boldsymbol{S}_{1}$ up to time $t=\tau$ and will switch from $\boldsymbol{S}_{1}$ to $\boldsymbol{S}_{2}$ when $t>\tau$ with minimal time. Notice that up to time $t=\tau$ this is both an output and an input constrained problem, once we must satisfy constrains (3) and (4); on the other hand, after $t>\tau$ we have an input constrained time-optimal problem. Therefore, we shall treat these two subproblems separately. We will find a control law denoted $u_{1, \tau_{-}}$for $t \leq \tau$, and another one denoted $u_{1, \tau_{+}}$for $t>\tau$.

For $t \leq \tau$ we must respect the constraints (3) and (4), but ti is not necessary that $y_{1}$ asymptotically tracks the reference. As long as the primary actuator respects the boundaries of the region $S_{1}$, we may use it in our behalf to optimize the transition time from an initial region $S_{1}$ to a given region $\boldsymbol{S}_{2}$. It is of our interest that, at $t=\tau$, the primary actuator is at the border of the region $S_{1}$ and with a large speed moving towards the region $\boldsymbol{S}_{2}$ (Fig. 2). During the design of the control law we shall assume, without loss of generality, that $r e f_{1}<r e f_{2}$.

In order to find $u_{1, \tau_{-}}(t)$ such that the above requirements are satisfied, we will take it to be a first order polynomial function. While this choice will provide us with a simple solution, higher order polynomials will not create a significant improvement. Also, we may justify the choice of a first order polynomial function for $u_{1, \tau_{-}}(t)$ by referring to the controller $u_{1, \tau_{0}}(t)$ as given in (7).

Proposition 3.2: Suppose the given parameters $p_{0}, v_{0}, p_{\tau}$ and $\tau$ satisfy

$$
\beta \geq 0, v_{0}>0, \beta:=4 \Delta p / \tau-v_{0}, \Delta p:=p_{\tau}-p_{0},
$$

and $\mathcal{U}_{1}$ and $\mathcal{U}_{2}$ satisfy

$$
\begin{aligned}
& \mathcal{U}_{1} \leq\left(-3 v_{0}+\sqrt{3 v_{0} \beta}\right) / \tau \\
& \mathcal{U}_{2} \geq\left(6 \Delta p / \tau-2 \sqrt{3 v_{0} \beta}\right) / \tau .
\end{aligned}
$$

(i) The set

$$
\begin{aligned}
\mathbb{K}:=\{k \in \mathbb{R}:|k| & \leq 1, k \geq\left(\tau \mathcal{U}_{1}+3 v_{0}\right) / \sqrt{3 v_{0} \beta}, \\
k & \left.\geq\left(3 \Delta p / \tau-\mathcal{U}_{2} \tau / 2\right) / \sqrt{3 v_{0} \beta}\right\}
\end{aligned}
$$

is not empty.

(ii) For any $k \in \mathbb{K}$, the controller

$$
u_{1, \tau_{-}}(t)=a t+b
$$


with

$$
\begin{aligned}
a & =6 \Delta p / \tau^{3}-6 v_{0} / \tau^{2}-3 b / \tau \\
b & =-3 v_{0} / \tau+k \sqrt{3 v_{0} \beta} / \tau
\end{aligned}
$$

drives the primary actuator from $x_{1}(0)=\left[\begin{array}{ll}p_{0} & v_{0}\end{array}\right]^{\top}$ to $x_{1}(\tau)=\left[\begin{array}{ll}p_{\tau} & v_{\tau}\end{array}\right]^{\top}$ for some $v_{\tau}>0$ while respecting the constraints (3) and (4).

(iii) For any $k \in \mathbb{K}$, we define the corresponding final velocity as $v_{\tau}(k)$. Then, $v_{\tau}\left(k^{*}\right) \geq v_{\tau}(k), \forall k \in \mathbb{K}$ for $k^{*}=\inf \mathbb{K}$.

Proof: (i) It suffices to prove

$$
\begin{array}{r}
1 \geq\left(\tau \mathcal{U}_{1}+3 v_{0}\right) / \sqrt{3 v_{0} \beta}, \\
1 \geq\left(3 \Delta p / \tau-\mathcal{U}_{2} \tau / 2\right) / \sqrt{3 v_{0} \beta}
\end{array}
$$

which are true under the assumptions (13) on $\mathcal{U}_{1}$ and $\mathcal{U}_{2}$.

(ii) With the given first order polynomial $u_{1, \tau_{-}}(t)$, we have

$$
\begin{aligned}
& p(t)=a t^{3} / 6+b t^{2} / 2+v_{0} t+p_{0} \\
& v(t)=a t^{2} / 2+b t+v_{0}
\end{aligned}
$$

by noting $p(0)=p_{0}$ and $v(0)=v_{0}$. For the $a, b$ given in (15), it is easy to show $p(\tau)=p_{\tau}$ and $v(\tau)>0$.

It is left to show that the constraints (3) and (4) are satisfied. For (4), we would like to have,

$$
p_{0} \leq p(t)=a t^{3} / 6+b t^{2} / 2+v_{0} t+p_{0} \leq p_{\tau}
$$

which is equivalent to

$$
0 \leq g(t):=a t^{3} / 6+b t^{2} / 2+v_{0} t \leq \Delta p .
$$

By noting $g(0)=0$ and $g(\tau)=\Delta p>0$, it suffices to show that $a$ and $b$ in (15) are such that $g(t)$ is monotonically increasing, i.e.,

$$
\dot{g}(t)=v(t) \geq 0 .
$$

As $v(t)$ is quadratic in $t$ and $v(t) \geq 0$ can be satisfied if the parabola is facing up $(a \geq 0)$ and if it has at the most one zero $\left(b^{2}-2 a v_{0} \leq 0\right)$. Manipulating these inequalities and noting $g(\tau)=\Delta p$, we have

$$
a=6 \Delta p / \tau^{3}-6 v_{0} / \tau^{2}-3 b / \tau \geq b^{2} /\left(2 v_{0}\right) .
$$

With the $b$ given in (15), the inequality (16) is satisfied for $|k| \leq 1$, i.e., for $k \in \mathbb{K}$.

For (3), it suffices to show

$$
\begin{aligned}
& u_{1, \tau_{-}}(0)=b \geq \mathcal{U}_{1} \\
& u_{1, \tau_{-}}(\tau)=a \tau+b \leq \mathcal{U}_{2}
\end{aligned}
$$

or equivalently,

$$
\begin{aligned}
-3 v_{0} / \tau+k \sqrt{3 v_{0} \beta} / \tau & \geq \mathcal{U}_{1} \\
6 \Delta p / \tau^{2}-2 k \sqrt{3 v_{0} \beta} / \tau & \leq \mathcal{U}_{2}
\end{aligned}
$$

which are true for $k \in \mathbb{K}$.

(iii) We note that

$$
\begin{aligned}
v(\tau) & =a \tau^{2} / 2+b \tau+v_{0} \\
& =3\left(\Delta p-v_{o} \tau\right) / \tau-b \tau / 2+v_{0} \\
& =3 \Delta p / \tau-v_{o} / 2-k \sqrt{3 v_{0} \beta} / 2
\end{aligned}
$$

and it becomes evident that to maximize $v(\tau)$ we must pick the smallest $k \in \mathbb{K}$. The proof is thus complete.

Remark 3.1: Generally there is a trade off between final velocity and input constraints. From the definition of $\mathbb{K}$, we see that for a smaller $\mathcal{U}_{1}$ and/or a larger $\mathcal{U}_{2}$, we may pick a smaller $k$. It means that for less constrains on the input, we may obtain a larger final velocity $v_{\tau}$.

After $t>\tau$, it becomes a pure time-optimal control problem under input constraints. Such problems are solved - by the well known bang-bang control strategy, a sliding mode controller that switches from $u_{1}=\mathcal{U}_{1}$ to $u_{1}=\mathcal{U}_{2}$ (In practice, we usually have $-\mathcal{U}_{1}=\mathcal{U}_{2}=u_{1 \max }>0$ ) according to a given switching curve. Despite its theoretical simplicity it is extremely hard to successfully implement such controller due to fact that imperfections in switching devices, model uncertainties and time delays cause bangbang controllers to suffer from chattering [13]. To avoid control chattering and its undesired consequences one may limit the number of switches the controller is allowed to perform to be at the most $n-1$ [14]. However, due to model uncertainties, plant disturbance and measurement noise, this will cause errors between the reference and the output when the switchings are over, hence, this form of controller will not asymptotically converge the system output to the reference point. However, the bang-bang controller may be applied to the primary actuator of a DSA system because there is no need for the primary to asymptotically track the reference. We may, therefore, use the bang-bang to drive the output $y_{1}$ from the manifold $S_{1}$ to the manifold $S_{2}$ by switching the controller only once. Then, as $y_{1}$ enters $\boldsymbol{S}_{2}$, we find ourselves in the next round of the control problem for $0 \leq t \leq \tau$.

Proposition 3.3: The controller $u_{1, \tau_{+}}$that achieves the transition of the primary actuator output $y_{1}$ from $\boldsymbol{S}_{1}\left(y_{1}=\right.$ $\left.r e f_{1}+r\right)$ to $\boldsymbol{S}_{2}\left(y_{1}=r e f_{2}-r\right)$ in minimum time is given by,

$$
u_{1, \tau_{+}}=\left\{\begin{array}{cll}
-u_{1 \max }, & \text { if } & y_{1}>-\frac{1}{2} v|v| \\
& \text { or if } y_{1}=-\frac{1}{2} v|v| \text { and } y_{1}<0 \\
u_{1 \max }, & \text { if } & y_{1}<-\frac{1}{2} v|v| \\
& \text { or if } \quad y_{1}=-\frac{1}{2} v|v| \text { and } y_{1}>0
\end{array}\right.
$$

Proof: The bang-bang control for double integrator systems is a classical problem in optimal control, for a proof please refer to [15].

\section{B. Secondary Actuator Control Design}

The goal of the control design for the secondary actuator is to enable $y_{2}$ in (1) to reduce the error generated between the reference and $y_{1}$. This error exists because the primary actuator moves freely in $\boldsymbol{S}_{i}$. Notice, however, that due to the construction of the manifold $\boldsymbol{S}_{i}$, as long as the primary stays in this manifold the secondary is able to reach the reference and compensate the error. In order to achieve this goal we propose the feedback/feedforward scheme presented in Fig. 4. Once the secondary actuator has a faster dynamics than 
the primary, it may be modeled as a simple gain $\left(-a_{1} / b_{2}\right)$ as seen in the figure; also, the gain $K=\left[k_{1} k_{2}\right]$ is computed so that the secondary actuator is highly damped and has a large bandwidth.

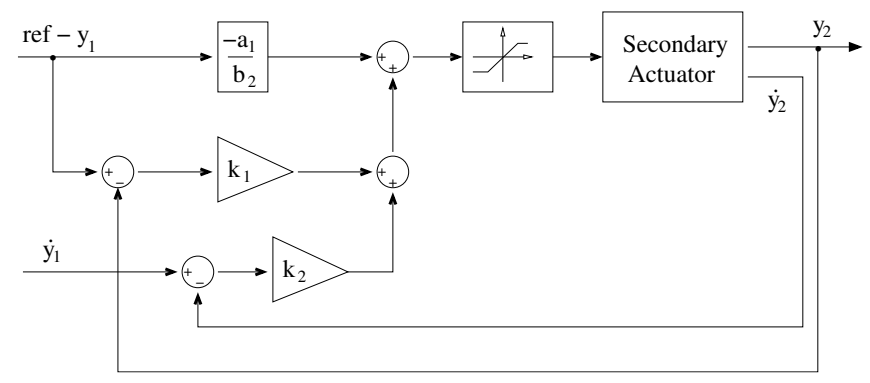

Fig. 4. Block diagram of the secondary actuator control.

Notice that when the primary actuator is moving from one manifold to another the reference will be out of reach of the secondary actuator, and, in such case, it will saturate. We allow this saturation to happen because, due to assumption $\mathrm{A} 1$, as soon as the primary actuator enters the next manifold $S_{2}$ the secondary actuator is able to follow the error generated by the primary and reach the reference point.

\section{An Illustrative Example}

Consider a DSA system described in (1) with the following parameters,

$$
\begin{array}{ll}
a_{1}=-5.0 \times 10^{7}, & b_{1}=2.0 \times 10^{7}, \\
a_{2}=-7.1 \times 10^{3}, & b_{2}=1.6 \times 10^{8} .
\end{array}
$$

We suppose that the saturation of the primary actuator occurs at $\left|u_{1}\right| \leq 1 \mathrm{~V}$, and for the secondary actuator $\left|u_{2}\right| \leq 3.5 \mathrm{~V}$. Furthermore, the range of actuation of the secondary actuator is considered to be $\pm 10 \mu \mathrm{m}$.

In order to compare the proposed methodology with the conventional one, where the primary actuator is asymptotically driven to the reference point, we have designed a linear feedback gain $K_{c}$ as follows,

$$
K_{c}=\frac{1}{b_{1}}\left[4 \pi^{2} \omega_{1}^{2} 4 \pi \omega_{1} \zeta_{1}\right]
$$

where $\zeta_{1}$ and $\omega_{1}$ represent the damping ratio and the undamped natural frequency of the closed-loop system $C_{1}(s I-$ $\left.A+B_{1} K_{c}\right)^{-1} B_{1}$, whose poles are at $2 \pi \omega_{1}\left(-\zeta_{1} \pm j \sqrt{1-\zeta_{1}^{2}}\right)$. We have chosen $\omega_{1}=30 \mathrm{~Hz}$ and $\zeta_{1}=0.9$ so that the primary actuator is tuned to have no overshoot. The secondary control design is the same for both the proposed and the conventional methodology. The gain $K=\left[\begin{array}{ll}k_{1} & k_{2}\end{array}\right]$ in Fig. 4 was designed such that the secondary system closed loop system has an undamped natural frequency of $\omega_{2}=400 \mathrm{~Hz}$ and a damping ratio of $\zeta_{2}=0.9$.

Two different simulation were performed: a first one for a sequence of large steps, such that the successive manifolds $S_{i}$ do not intersect each other and we must apply the control $u_{1, \tau_{-}}$followed by $u_{1, \tau_{+}}$; and another for a sequence of steps within the range of the secondary actuator, where we apply $u_{1, \tau_{0}}$. During these simulations we define the settling time to be the time that it takes for the total position output $y$ to enter and remain within $\pm 1 \mu \mathrm{m}$ relative to the setpoint.

1) $S_{1} \cap S_{2}=\varnothing$ : For the first simulation we suppose the system output must stay at the position $y=0 \mu \mathrm{m}$ for $20 \mathrm{~ms}$, then move to $y=50 \mu \mathrm{m}$ and stay there for another $20 \mathrm{~ms}$, and finalize at the position $y=100 \mu \mathrm{m}$. We may create a vector $P$ of positions to be tracked linked to another vector $\Gamma$ representing the time to be spent in each position, in this case,

$$
\begin{aligned}
& P_{1}=\left[\begin{array}{rcc}
0 & 50 & 100
\end{array}\right], \quad[\mu m], \\
& \Gamma_{1}=\left[\begin{array}{llcl}
20 & 20 & \infty
\end{array}\right], \quad[m s] .
\end{aligned}
$$

when we achieve the final position of $100 \mu \mathrm{m}$ we stabilize the primary actuator with the same linear gain used in the conventional form of control and wait for another vector of references.

Comparing the plots in Fig. 5 we notice the effectiveness of the proposed methodology. When moving from 0 to $50 \mu \mathrm{m}$, the conventional control requires $9 \mathrm{~ms}$, while the proposed one requires only $2.5 \mathrm{~ms}$ (an improvement of over $70 \%$ regarding settling time is achieved). Notice that the improvement is even greater when moving from 50 to 100 $\mu \mathrm{m}$, this is due to the fact that at $42.5 \mathrm{~ms}$ the primary actuator is able to leave its current manifold with a larger velocity then it did at $20 \mathrm{~ms}$.

2) $S_{2} \bigcap S_{2} \neq \varnothing$ : For this second simulation we suppose a series of steps within the range of the secondary actuator such that the control law $u_{1, \tau_{0}}$ must be applied. In this case,

$$
\begin{aligned}
& P_{2}=\left[\begin{array}{llllllll}
0 & -5 & 10 & 25 & 40 & 25 & 10 & 0
\end{array}\right],[\mu m], \\
& \Gamma_{2}=\left[\begin{array}{llllllll}
10 & 10 & 10 & 10 & 20 & 10 & 10 & \infty
\end{array}\right], \quad[m s] .
\end{aligned}
$$

Notice how in the proposed strategy we position $y_{1}$ (thin solid line) in between the manifolds, this avoids the saturation of the secondary actuator and allows it to reach the reference in a smaller time (Fig. 6).

\section{CONClusion And Future WORK}

In this paper we have proposed a form of "preview control" for DSA systems where the information of future reference points is used. While in the conventional approach the primary actuator is designed to asymptotically track the reference points, the proposed methodology accounts for the redundancy of actuators and allows the primary actuator to move freely inside a manifold $\boldsymbol{S}$. This manifold is determined by the range of the secondary actuator in such a way that when the primary actuator is in $S$, the secondary actuator is able to reach the reference point and, thus, compensate the error between the primary actuator and the reference. The main contribution of this paper is taking advantage of the freedom the primary actuator has inside the manifold $S$ in order to optimize the transition between the present reference point and the immediate future one. A numerical example demonstrates the effectiveness of the proposed control design over the conventional one.

While three different controllers were designed for the primary actuator, namely, $u_{1, \tau_{-}}, u_{1, \tau_{+}}$and $u_{1, \tau_{0}}$, we observed that $u_{1, \tau_{-}}$is a somewhat conservative solution, once 

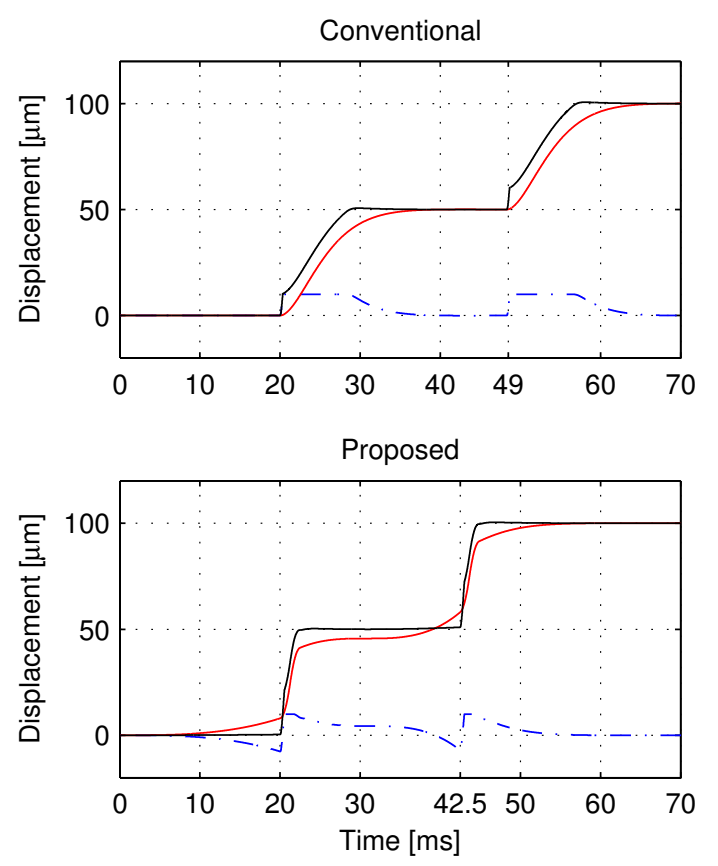

Fig. 5. Simulation result showing the effectiveness of the proposed control strategy. Thick solid line: total output; thin solid line: primary actuator output; dash-dotted line: secondary actuator output.

it is based the monotonicity of the primary actuator, and, despite the simulation results showing that $u_{1, \tau_{0}}$ respects the boundaries of the manifolds, there is no mathematical proof that such constraint is satisfied. Therefore, future works will focus on a unified solution combining $u_{1, \tau_{-}}$and $u_{1, \tau_{0}}$ that is less conservative and that guarantees the constraints will be satisfied.

\section{REFERENCES}

[1] K. Mori, T. Munemoto, H. Otsuki, Y. Yamaguchi, and K. Akagi, "A dual-stage magnetic disk drive actuator using a piezoelectric device for a high track density," IEEE Trans. Magn., vol. 27, no. 6, pp. 52985300, Nov. 1991.

[2] K.W. Chan, W.H. Liao, and I.Y. Shen, "Precision positioning of hard disk drives using piezoelectric actuators with passive damping," IEEE/ASME Trans. Mechatron., vol. 13, no. 1, pp. 147-151, Feb. 2008.

[3] B. Kim, J. Li, and T. Tsao, "Two-parameter robust repetitive control with application to a novel dual-stage actuator for noncircular machining," IEEE/ASME Trans. Mechatron., vol. 9, no. 4, pp. 644-652, Dec. 2004.

[4] A. Elfizy, G. Bone, and M. Elbestawi, "Design and control of a dualstage feed drive," Int. Journal of Machine Tools \& Manufacture, vol. 45, pp. 153-165, 2005.

[5] X. Huang, R. Horowitz, and Y. Li, "A comparative study of MEMS microactuators for use in a dual-stage servo with an instrumented suspension," IEEE/ASME Trans. Mechatron., vol. 11, no. 5, pp. 524532, Oct. 2006.
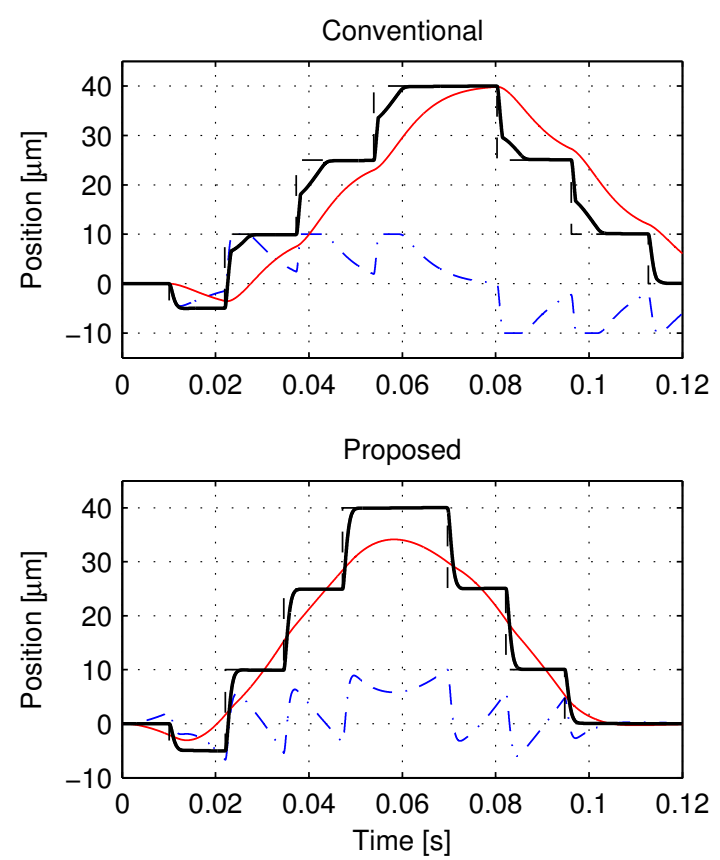

Fig. 6. Simulation results for a series of steps within the range of the secondary actuator.

[6] M. Kobayashi, and R. Horowitz, "Track seek control for hard disk dual-stage servo systems," IEEE Trans. Magn., vol. 37, no. 2, pp. 949-954, Mar. 2001.

[7] S. Lee, and Y. Kim, "Minimum destructive interference design of dualstage control systems for hard disk drives," IEEE Trans. Contr. syst. Technol., vol. 12, no. 4, pp. 517-531, Jul. 2004.

[8] B. Hredzak, G. Herrmann, and G. Guo, "A proximate-time-optimal control design and its application to a hard disk drive dual-stage actuator system," IEEE Trans. Magn., vol. 42, no. 6, pp. 1708-1715, Jun. 2006.

[9] J. Zheng, and M. Fu, "Nonlinear Feedback Control of a Dual-Stage Actuator System for Reduced Settling Time," IEEE Trans. Contr. Syst. Technol., vol. 16, no. 4, pp. 717-725, Jul. 2008.

[10] J. Zheng, M. Fu, Y. Wang, and C. Du, "Nonlinear tracking control for a hard disk drive dual-stage actuator system," IEEE/ASME Trans. Mechatron., vol. 13, no. 5, pp. 510-518, Oct. 2008.

[11] D. Iamratanakul, B. Jordan, K. K. Leang, and S. Devasia, "Optimal Output Transitions for Dual-Stage Systems," IEEE Trans. Contr. syst. Technol., vol. 16, no. 5, pp. 869-881, Sep. 2008.

[12] T. B. Sheridan, "Three models of preview control," IEEE Trans. Human Factors Electron., vol. HFE-7, no. 2, pp.91-102, Jun. 1966.

[13] H. K. Khalil, Nonlinear Systems, 3rd ed. Upper Saddle river, NJ: Prentice Hall, 2002.

[14] L. S. Pontryagin, V. G. Boltyanskii, R. V. Gamkrelidze, and E. F. Mishchenko, The mathematical theory of optimal processes. New York: Wiley-Interscience, 1962.

[15] A. E. Bryson, and Y. C. Ho, Applied Optimal Control. New York: Hemisphere, 1975. 